\title{
The role of epigenetics in spermatogenesis
}

\author{
Sezgin Güneş, Tuba Kulaç
}

Department of Medical Biology, Faculty of Medicine, Ondokuz Mayıs University, Samsun, Turkey

Submitted: 01.02 .2013

Accepted: 30.04.2013

Correspondence: Sezgin Güneş Department of Medical Biology, Faculty of Medicine, Ondokuz Mayıs University, 55139 Samsun, Turkey Phone: +90 36231219 19-3164 E-mail: sgunes@omu.edu.tr

(C) Copyright 2013 by Turkish Association of Urology

Available online at www.turkishjournalofurology.com

\begin{abstract}
Male germ cells have a unique morphology and function to facilitate fertilization. Sperm deoxyribonucleic acid (DNA) is highly condensed to protect the paternal genome during transfer from male to oocyte. Sperm cells undergo extensive epigenetic modifications during differentiation to become a mature spermatozoon. Epigenetic modifications, including DNA methylation, histone modifications, and chromatin remodeling are substantial regulators of spermatogenesis. DNA hypermethylation is associated with gene silencing. Meanwhile, hypomethylation is associated with gene expression. In sperm cells, promoters of developmental genes are highly hypomethylated. Proper DNA methylation is essential for embryo development. Histone modifications are chemical modifications that change the DNA-binding capacity of histones and the accessibility of regulatory factors to the DNA, thereby altering gene expression. Phosphorylation, methylation, acetylation, and ubiquitination are primary modifications of lysine and serine residues on histone tails. In addition to somatic histones, testis-specific histone variants are expressed, including histone $\mathrm{H} 2 \mathrm{~B}$ in mature sperm. The replacement of histones with protamines is a crucial step in spermatogenesis. Histone hyperacetylation induces a loose chromatin structure and facilitates topoisomerase-induced DNA strand breaks. As a result, histones are replaced with transition proteins. Next, the transition proteins are replaced with protamines that induce compaction of sperm DNA. This review provides an overview of epigenetic changes during spermatogenesis.
\end{abstract}

Key words: Chromatin remodeling; DNA methylation; epigenetics; histone modification; spermatogenesis.

Epigenetics is defined as stable changes which can be inherited through mitotic or meiotic division, but alter gene expression without modifying DNA sequences. ${ }^{[1]}$ In antique Greek epi-, prefix means over, above, and beyond. Therefore it means over, and beyond genetics.

Epigenetic mechanisms mediate interactions between DNA, and miscellaneous proteins which have regulatory roles in replication, and gene expression processes at various stages of development during the life of an organism, with DNA. Each cell type has a specific epigenetic signature. This signature reflects developmental history, and environmental effects in the phenotype of the cell, and the organism. $^{[2]}$

Epigenetic changes involve histone proteins which function in the packaging of DNA, and also a series of DNA modifications.DNA encircles histone proteins, and constitutes fundamental structural unit of the chromatin, namely nucleosome. It regulates DNA meth- ylation, and also via modifications of histone, and chromatin it also controls gene expression under varying temporal, and environmental conditions. ${ }^{[3,4]}$ During development epigenetic profile of the germ cells changes..$^{[5,6]}$ During post-implantation embryonic phase, pluripotent cells of epiblasts ensure development of primordial germ cells (PGCs). In women PGCs are suppressed during prophase of meiosis I, in men it enters into the phase of mitotic arrest. Epigenetic profiles of germ cells undergo transformations during different phases of meiotic division. ${ }^{[7]}$

In this article we will review methylation of sperm DNA, sperm histone, and chromatin modifications occurring during spermatogenesis.

\section{Sperm DNA methylation}

DNA methylation is a biochemical procedure where a methyl group is added to the 5. carbon of the pyrimidine ring of the cytosine nucleotide located in the $\mathrm{CpG}$ islands ${ }^{[8]} \mathrm{CpG}$ islands 
are 500 bp long-genomic regions which contain very condensed CG dinucleotides (CG/GC >55\%). ${ }^{[9]}$ These sequences are found in the promoter regions of nearly $40 \%$ of mammalian genes. DNA methylation seen in $\mathrm{CpG}$ islads in the promoter region leads to inheritable silencing at the transciption level. DNA methylation seen in $\mathrm{CpG}$ islands plays an important role in the regulation of gene expression. In the regulation of gene expression, methylation especially in the promoter regions of genes, induces changes in the recognition regions of transcription factors with resultant prevention of these factors. This process plays a role in the suppression of gene expression. ${ }^{[10]}$

DNA methylation occurs under the impact of DNA methyltransferase (DNMT) enzyme. One of DNA methyltransferases [one DNMT1, one DNMT2, three DNMT3 (DNMT3a/3b and DNMT3L)] catalyze transfer of one methyl group from S-adenosyl methionine to cytosine. ${ }^{[10]}$ DNMT1 is responsible from maintenance of methylation motif during DNA replication, and termed as maintenance methyltransferase. DNMT3s mediate de novo methylation of unmethylated cytosines, and methylates genomic DNA during early phase of embryonic development. Though the role of DNMT2 has not been fully elucidated, as suggested by researchers, it might exert an impact as a tRNA methyltransferase. ${ }^{[10-12]}$

These acquired changes are perpetual, and permanent. ${ }^{[4,13]}$ Hypo-, and hypermethylation can occur spontaneously in different regions of the genome. ${ }^{[14]}$

In a recent study, sperm methylation maps of human beings, and chimpanzees have been determined. In this study, it was reported that promoters of the genes responsible from development were hypomethylated, and manifested stronger hypomethylation when compared with promoters of the somatic cells. Besides, recurrent regions of the sperm genome reportedly demonstrate high degrees of methylation, while transposons manifest weaker methylation. ${ }^{[15]}$

\section{Sperm Histone Modification}

Histones are basic proteins rich in lysine, and arginine. They are located in the nuclei of eukaryotic cells, and induce packaging of DNA within lysosomes. $\mathrm{H} 2 \mathrm{~A}, \mathrm{H} 2 \mathrm{~B}, \mathrm{H} 3$, and $\mathrm{H} 4$ histone proteins are found in the nuclei of nucleosomes. Nucleosomes contain octameric proteins formed by combination of two $\mathrm{H} 3-\mathrm{H} 4$ dimers, and two H2A-H2B dimers, and they are bound by $\mathrm{H} 1$ histone proteins. ${ }^{[16]}$ Via simple chemical modifications, histones change DNA -binding capacities, and interaction of other regulatory factors with DNA, and thus lead to alterations in gene activation. Histone modifications are linked to specific enzymes. ${ }^{[17]}$ Histone modifications are realized by acetylation, methylation, phosphorylation, and ubiquitination. Generally, acetylation of histone $\mathrm{H} 3$, and $\mathrm{H} 4$ leads to open chromatin configuration, and active transcription which facilitates binding of transcription factors. ${ }^{[18]}$ However deacetylation is related to inactivation of transcription, and generally correlates with methylation. Generally, histone acetylation is seen in regions of active transcription, hypoacetylated histones are localized in euchromatic, and heterochromatic regions. ${ }^{[19]}$ Another regulatory mechanism is methylation of lysine found in histone tails. Chemical changes as conversion of lysine in histone tails to serine are realized through methylation, acetylation and ubiquitination. ${ }^{[20,21]} \mathrm{H} 3 \mathrm{~K} 9$, and $\mathrm{H} 3 \mathrm{~K} 27$ histones are generally associated with inactivation. ${ }^{[2]}$ However methylation of histones take place both in active, and inactive chromatin sites. Methylation of 9. lysine on amino terminal of histone (H3-9K) leads to DNA silencing, and also involves heterochromatic regions. On the other hand, methylation of the 4. lysine of $\mathrm{H} 3$ protein (H3-4K) is associated with activation, and mainly it takes place in promoter regions of active genes. During spermatogenesis, methylation of histone tails is achieved by H3-K4, and H3-K9 methyltransferases. ${ }^{[23]}$

Modifications between $\mathrm{H} 3$ and $\mathrm{H} 4$ histone protein tails lead to various interactions. Some of them regulate transitions between active, and inactive chromatin states through a reverse mechanism called histone code. ${ }^{[24]}$

Premeiotic PGCs, and spermatogonia demonstrate specific histone H3K9me3 modification model. ${ }^{[25,26]}$ However, with the onset of meiotic process in male germ cells these models change (Figure 1). ${ }^{[27]}$ Histone modification, and changes in its composition play important roles in chromatin modifications required for normal meiotic process, and later maturation of gametes. ${ }^{[6]}$ Both female, and male gamete cells undergo developmental changes following termination of meiotic division. Some histones on X chromosome, label, and retain $\mathrm{H} 3 \mathrm{~K} 9 \mathrm{me} 2$, global remodelling in haploid spermatids occur at a lower rate. [28,29] Variants of $\mathrm{H} 2 \mathrm{~A}, \mathrm{H} 2 \mathrm{~B}, \mathrm{H} 3$, and $\mathrm{H} 1$ are expressed in testis. Compared with standard histones, histone variants have a lower degrees of stability. ${ }^{[30]}$ In testis, in addition to testis-specific histone variants, somatic histone variants are also expressed. In mature sperm, the most frequently testis-specific histone $\mathrm{H} 2 \mathrm{~B}$ is found. ${ }^{[31]}$ Ion channels, and genes involving spermatogenesis are rich in testis-specific histone $\mathrm{H} 2 \mathrm{~B}$ contrary to genes responsible from development. Testis-specific H1T2- binding histone variant has an important role in the condensation of chromatin during spermatogenesis. ${ }^{[32]}$ As demonstrated in immunohistochemical studies, histone variant $\mathrm{H} 2 \mathrm{AZ}$ which is found in some certain cell types condenses in pericentromeric regions of hemochromatins. ${ }^{[33,34]} \mathrm{H} 3 \mathrm{~K} 4 \mathrm{me} 3$ condenses in genes involving 
in spermatogenesis, while $\mathrm{H} 3 \mathrm{~K} 4 \mathrm{me} 2$ is found in regions rich in developmental genes. Hyperacetylation of histone $\mathrm{H} 4$ is responsible from replacement of histones with protamines in haploid spermatids.. HI1s1-binding histone variant (histone-1-like protein in spermatids 1 ) is expressed in elongated spermatids. ${ }^{[35,36]}$ As a consequence, histone packaging is an evolutional, and developmental procedure for spermatogenesis. ${ }^{[33]}$

These two epigenetic regulatory mechanisms, ie. DNA methylation, and histone modification are closely related to each other in the process of gene expression. Successful regulation, and control of gene expression requires close cooperation, and interaction of both of these mechanisms. ${ }^{[1]}$

\section{Modification of chromatin during spermatogenesis}

Fertilization requires realization of many physiological events including movement of sperm cells all along the female reproductive system, their attachment to zona pellucida, and penetration into oocyte. ${ }^{[37]}$ For the accomplishment of all these phases, a regulatory mechanism involving striking modifications which require replacement of $90-95 \%$ histones by protamines becomes effective. ${ }^{[38]}$ Protamines are small molecules rich in arginine. They are located in nuclei, and synthetized during advanced stages of spermatogenesis. ${ }^{[39]}$ Protamination of sperm chromatin facilitates compaction of nucleus required for sperm motility, and also protects sperm genome from oxidation, and harmful molecules within the female reproductive system. Extensive packaging of DNA following protamination prevent transcription. Protamination is an epigenetic regulatory process specific to sperm cells..$^{[38]}$ Before fertilization, paternal haploid genome carried by mature sperm is packaged tightly by protamines, maternal genome which is suppressed in metaphase II is packaged by histone proteins. After fertilization, protamines are rapidly translocated with histones, and oocyte passes through metaphase II, and polar body is thrown out. H3, and H4 histones found in paternal chromatin are more effectively acetylated when compared with those contained in maternal chromatin. ${ }^{[40,41]}$

Translocation mechanism taking place between histone proteins, and protamines which ensures packaging of sperm DNA is still a partially understood multistep process Early modification processes involve replacement of histone variants by selected histones which are expressed during spermatogenesis. In mature sperm mostly testis-specific histone $2 \mathrm{~B}$ protein variant is found which attracts attention because of its unique expression characteristic. Characteristic in that it does not undergo polyadenilation at its 3' terminal, and it is localized in telomeres of sperm chromosomes. ${ }^{[42]}$ Acetylation of some histones accelerates due to their replacement by their variants.

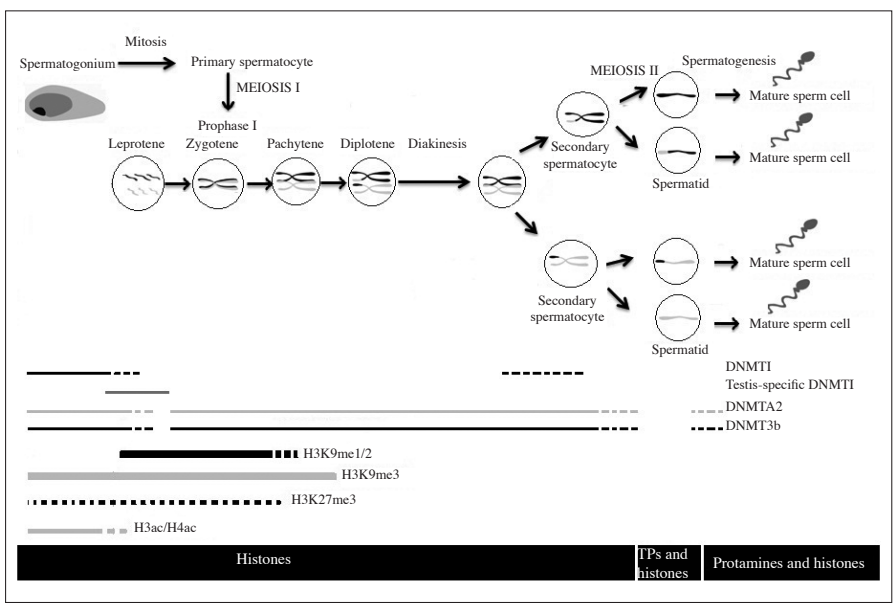

Figure 1. Spermatogenesis, and epigenetic changes during spermatogenesis

Acetylation is regulated by acetylase, and deacetylase enzymes. Hyperacetylation of histones leads to loosening of chromatin structure. Loose protein structure stimulates DNA strand breaks caused by topoisomerase enzyme, and facilitates separation of histones, and their replacement by transition proteins (TPs).

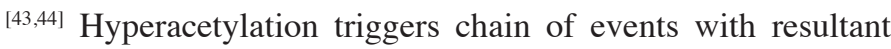
replacement of histones by protamines. ${ }^{[45]}$ Transition proteins 1 , and 2 demonstrate moderately basic non-histone proteins. They involve in 12., and 13. stages of spermatogenesis, but leave the process at 14 . stage. ${ }^{[30]}$ These proteins stick to DNA, and thus play critical roles in separation of histone variants, and later condensation by protamine. ${ }^{[46]}$ Then TPs are totally replaced by protamines. Two protamines (P1, and P2) are nearly equally expressed in human beings. TP, and protamine expressed genes are considerably overlap (Figure 2). ${ }^{[47]}$ These also have a unique translational regulatory mechanism. ${ }^{[48]}$ This important posttranslational mechanism involves phosphorylation required for appropriate chromatin condensation. ${ }^{[49]}$ In mice where one of these transition proteins was intentionally mutated (knockout mice), despite DNA-packaging, though inadequate, could be made with the aid of the other transition protein. and protamine with resultant infertile mice. ${ }^{[50]}$ Histones break after their replacement by protamine. Incorporation of protamines into sperm chromatin induces tighter DNA-packaging. Thus during configuration, and transfer of spermatozoa, safe conditions are created for the genome. Oakes et al. ${ }^{[51]}$ reported that the characteristic features of DNA methylation change only a little bit during evolution of genome after pachytene stage of spermatocytes. In fertile men, ratio of $\mathrm{P} 1 / \mathrm{P} 2$ protamines equal to one. Erroneous processing of protamine transcripts leads to increase in the production of immature P2 precursors associated with subfertility. ${ }^{[52,53]}$ Subfertility is also associated with derangement of $\mathrm{P} 1 / \mathrm{P} 2$ ratio. ${ }^{[38,54]}$ Despite controversial publica- 


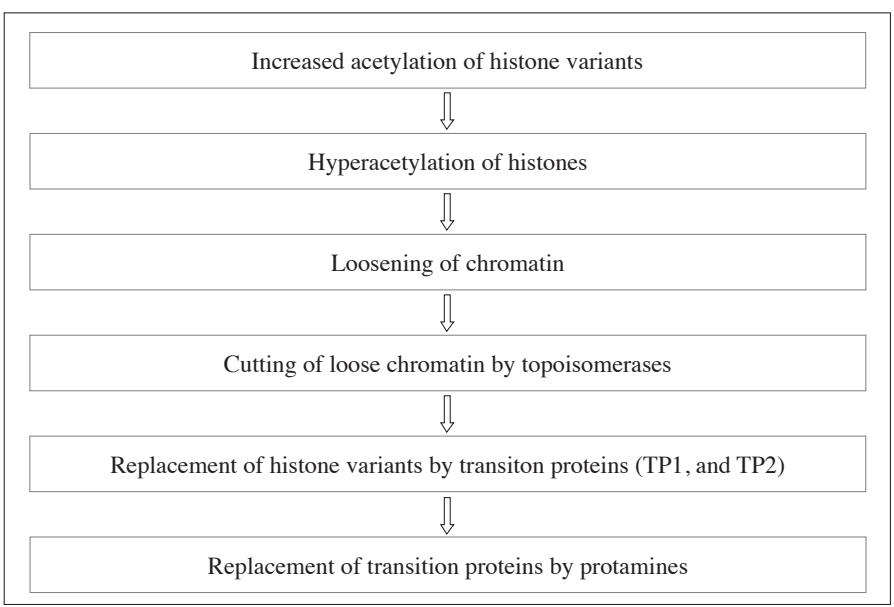

Figure 2. Replacement of histones localized in sperm chromatin by protamines

tions, deviations in protamine ratio has been associated with various phenotypic features including decreased sperm counts, and function, and poor embryonic quality. ${ }^{[48,55,56]}$ Besides variations were detected in average $\mathrm{P} 1 / \mathrm{P} 2$ ratio in ejaculate at different measurement time points. ${ }^{[57,58]}$ In conclusion, abnormal protamination is seen prevalently in men with abnormal sperm parameters contrary to fertile men with normal sperm parameters. ${ }^{[53,59]}$ In many studies abnormal replacement of histones by protamines have been associated with reduced semen quality, decreased success rates of IVF, and pregnancy rates. ${ }^{[56,58,60,61]}$

Appropriate protamine levels cause firmly packaging of chromatin leading to tighter compaction of sperm nuclei. Mammalian sperm chromatin constitutes of three parts. These components include protamine-bound DNA, histone-bound DNA, and sperm nuclear matrix bound-DNA. ${ }^{[62]}$ Packaging of DNA with protamine is realized via formation of disulfide bonds between protamines, and toroidal structure of the chromatin subunit.

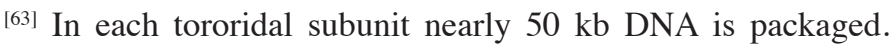
[39] Recently Ward has proposed a specific model of DNApackaging by protamines. According to this donut loop model, each toroid links, loop domains of DNA and matrix attachment regions. ${ }^{[62,64]}$ This model demonstrates that protamine-bound DNA is protected from damage, by compressing, and packing toroids, while toroid-linker, and histone-bound DNA regions are exposed to DNA damage induced by endonucleases. ${ }^{[65]}$

On the other hand, nearly 5-10\% of DNAs of fertile, and a higher percentage of DNAs in infertile men are bound to histones. ${ }^{[66,67]}$ It has been reported that the presence of somatic cell like-chromatins in the sperm nuclei can induce transmission of divergent genetic information to offsprings. Up to recent times, we were ignorant about the function of histones which translo- cate with protamines in the sperm nuclei, and mostly inadequate protaminations were thought to be the source of these histones. Two important studies have determined the location of histones which do not replace with protamines, and also retained all along the sperm genome. Arpanahi et al. cut human, and rat sperm chromatin with endonucleases, and determined endonucleasesensitive regions. ${ }^{[68]}$ They reported that endonuclease-sensitive regions are mainly bound to histones, and harbour regulatory genomic regions which also contain promoters. Hammoud et al. ${ }^{[66]}$ used a different method. They used micrococcal nuclease digestion, and gel purification procedures to differentiate chromatin-bound histone from protamine-bound histone. This protein purification is followed by microarray analysis, and sequencing procedure so as to identify histone-bound DNA, and protein-bound DNA. In this study, sperms of fertile, and normospermic men were used. It has been shown that histones concentrate in the promoters of required genes to ensure their development, and this process is not interrupted in miRNA, and genes undergoing imprinting. This study demonstrates that protamines do not concentrate in any gene family. In conclusion, these two studies demonstrate that the retained histones might have a potential epigenetic role in the embryonic development. As reported by Hammoud et al. epigenetic role of the variants of retained histones might be directed to a certain goal. Indeed, their more recent studies have demonstrated that both genes responsible for the development of embryonic stem cells, and $\mathrm{H} 3 \mathrm{~K} 4 \mathrm{me} 3$ and $\mathrm{H} 3 \mathrm{~K} 27 \mathrm{me} 3$ in critical genes responsible for the development were bivalently marked. These bivalently marked DNA regions are also methylated. They are also situated between regions which ensure creation of balanced state for genome. ${ }^{[6,69]}$ Recent studies indicate that histones have an important role in the determination of the epigenetic position of the sperm. They do not distribute in the genome randomly, and specific histone modifications regulate activation, and silencing of the genome. [69-71] These data have shown that sperm chromatin can transfer inheritable epigenetic characteristics which might exert an effect on post-fertilization transcriptional regulation. ${ }^{[72]}$

Despite numerous studies performed on male infertility within last years, still substantial number of cases of infertility remain unexplained. Various studies have demonstrated critical roles of epigenetic factors in spermatogenesis. Thorough understanding of these factors, and their mechanisms of action may contribute to the determination of the causes of male infertility, and also ways of controlling these mechanisms.

Author Contributions: Concept - S.G., T.K.; Design - S.G., T.K.; Supervision - S.G.; Funding - S.G.; Materials - S.G., T.K.; Data Collection and/or Processing - S.G., T.K.; Analysis and/or Interpretation - S.G., T.K.; Literature Review - S.G., T.K.; Writer - S.G., T.K.; Critical Review - S.G. 
Acknowledgements: We would like to thank Mert Nahir for drawing of figure.

Conflict of Interest: No conflict of interest was declared by the authors.

Financial Disclosure: The authors declared that this study has received no financial support.

\section{References}

1. Carrell DT. Epigenetics of the male gamete. Fertil Steril 2012;97:267-74.

2. Angers B, Castonguay E, Massicotte R. Environmentally induced phenotypes and DNA methylation: how to deal with unpredictable conditions until the next generation and after. Mol Ecol 2010;19:1283-95.

3. Li E. Chromatin modification and epigenetic reprogramming in mammalian development. Nat Rev Genet 2002;3:662-73.

4. Richardson BC. Role of DNA methylation in the regulation of cell function autoimmunity, aging and cancer. J Nutr 2002;132:24015 .

5. Surani MA, Hayashi K, Hajkova P. Genetic and epigenetic regulators of pluripotency. Cell 2007;128:747-62.

6. Kimmins S, Sassone-Corsi P. Chromatin remodelling and epigenetic features of germ cells. Nature 2005;434:583-9.

7. Dada R, Kumar M, Jesudasan R, Fernández JL, Gosálvez J, Agarwal A. Epigenetics and its role in male infertility. J Assist Reprod Genet 2012;29:213-23.

8. Talbert PB, Henikoff S. Spreading of silent chromatin: inaction at a distance. Nat Rev Genet 2006;7:793-803.

9. Takai D, Jones PA. Comprehensive analysis of $\mathrm{CpG}$ islands in human chromosomes 21 and 22. Proc Natl Acad Sci U S A 2002;99:3740-5.

10. Yi SV, Goodisman MA. Computational approaches for understanding the evolution of DNA methylation in animals. Epigenetics 2009;4:551-6.

11. Okano M, Xie S, Li E. Cloning and characterization of a family of novel mammalian DNA (cytosine-5) methyltransferases. Nat Genet 1998;19:219-20.

12. Gabor Miklos GL, Maleszka R. Epigenomic communication systems in humans and honey bees: from molecules to behavior. Horm Behav 2011;59:399-406.

13. Wilson VL, Jones PA. DNA methylation decreases in aging but not in immortal cells. Science 1983;220:1055-7.

14. Issa JP. CpG-island methylation in aging and cancer. Curr Top Microbiol Immunol 2000;249:101-18.

15. Molaro A, Hodges E, Fang F, Song Q, McCombie WR, Hannon GJ, et al. Sperm methylation profiles reveal features of epigenetic inheritance and evolution in primates. Cell 2011;146:1029-41.

16. Campos EI, Reinberg D. Histones: annotating chromatin. Annu Rev Genet 2009;43:559-99.

17. Cairns BR. The logic of chromatin architecture and remodelling at promoters. Nature 2009;461:193-8.
18. Liu Y, Lu C, Yang Y, Fan Y, Yang R, Liu CF, et al. Influence of histone tails and $\mathrm{H} 4$ tail acetylations on nucleosome-nucleosome interactions. J Mol Biol 2011;14:749-64.

19. Peng L, Seto E. Deacetylation of nonhistone proteins by HDACs and the implications in cancer. Handb Exp Pharmacol 2011;206:3956.

20. Kouzarides T. Chromatin modifications and their function. Cell 2007;128:693-705.

21. Handy DE, Castro R, Loscalzo J. Epigenetic modifications: basic mechanisms and role in cardiovascular disease. Circulation 2011;123:2145-56

22. Werner M, Ruthenburg AJ. The united states of histone ubiquitylation and methylation. Mol Cell 2011;43:5-7.

23. Carrell DT, Emery BR, Hammoud S. The aetiology of sperm protamine abnormalities and their potential impact on the sperm epigenome. Int J Androl 2008;31:537-45.

24. Lachner M, O’Sullivan RJ, Jenuwein T. An epigenetic road map for histone lysine methylation. Cell Sci 2003;116:2117-24.

25. McLaren A. Primordial germ cells in the mouse. Dev Biol 2003;262:1-15.

26. Payne C, Braun RE. Histone lysine trimethylation exhibits a distinct perinuclear distribution in Plzf-expressing spermatogonia. Dev Biol 2006;293:461-72.

27. Peters AH, O'Carroll D, Scherthan H, Mechtler K, Sauer S, Schofer C, et al. Loss of the Suv39h histone methyltransferases impairs mammalian heterochromatin and genome stability. Cell 2001;107:323-37

28. Namekawa SH, Park PJ, Zhang LF, Shima JE, McCarrey JR, Griswold MD, et al. Postmeiotic sex chromatin in the male germline of mice. Curr Biol 2006;16:660-7.

29. Turner JM, Mahadevaiah SK, Ellis PJ, Mitchell MJ, Burgoyne PS. Pachytene asynapsis drives meiotic sex chromosome inactivation and leads to substantial postmeiotic repression in spermatids. Dev Cell 2006;10:521-9.

30. Gaucher J, Reynoird N, Montellier E, Boussouar F, Rousseaux S, Khochbin S. From meiosis to postmeiotic events: the secrets of histone disappearance. FEBS J 2010;277:599-604.

31. van Roijen HJ, Ooms MP, Spaargaren MC, Baarends WM, Weber RF, Grootegoed JA, et al. Immunoexpression of testis-specific histone $2 \mathrm{~b}$ in human spermatozoa and testis tissue. Hum Reprod 1998;13:1559-66.

32. Martianov I, Brancorsini S, Catena R, Gansmuller A, Kotaja N, Parvinen $\mathrm{M}$, et al. Polar nuclear localization of H1T2, a histone H1 variant, required for spermatid elongation and DNA condensation during spermiogenesis. Proc Natl Acad Sci U S A 2005;102:2808-13.

33. Carrell DT, Hammoud SS. The human sperm epigenome and its potential role in embryonic development. Mol Hum Reprod 2010;16:37-47.

34. Rangasamy D, Berven L, Ridgway P, Tremethick DJ. Pericentric heterochromatin becomes enriched with H2a.Z during early mammalian development. EMBO J 2003;22:1599-607.

35. Meistrich ML, Trostle-Weige PK, Lin R, Bhatnagar YM, Allis CD. Highly acetylated $\mathrm{H} 4$ is associated with histone displacement in rat spermatids. Mol Reprod Dev 1992;31:170-81. 
36. Sonnack V, Failing K, Bergmann M, Steger K. Expression of hyperacetylated histone $\mathrm{H} 4$ during normal and impaired human spermatogenesis. Andrologia 2002;34:384-90.

37. Yamauchi Y, Shaman JA, Ward WS. Non-genetic contributions of the sperm nucleus to embryonic development. Asian J Androl 2011;13:31-5.

38. Oliva R. Protamines and male infertility. Hum Reprod Update 2006;12:417-35.

39. Balhorn R. A model for the structure of chromatin in mammalian sperm. J Cell Biol 1982;93:298-305.

40. Santos F, Hendrich B, ReikW, DeanW. Dynamic reprogramming of DNA methylation in the early mouse embryo. Dev Biol 2002;241:172-82.

41. Adenot PG, Mercier Y, Renard JP, Thompson EM. Differential $\mathrm{H} 4$ acetylation of paternal and maternal chromatin precedes DNA replication and differential transcriptional activity in pronuclei of 1-cell mouse embryos. Development 1997;124:4615-25.

42. Churikov D, Siino J, Svetlova M, Zhang K, Gineitis A, Morton Bradbury E, et al. Novel human testis-specific histone H2B encoded by the interrupted gene on the $\mathrm{X}$ chromosome. Genomics 2004;84:745-56.

43. Rousseaux S, Boussouar F, Gaucher J, Reynoird N, Montellier E, Curtet S, et al. Molecular models for post-meiotic male genome reprogramming. Syst Biol Reprod Med 2011;57:50-3.

44. Song N, Liu J, An S, Nishino T, Hishikawa Y, Koji T. Immunohistochemical analysis of histone $\mathrm{H} 3$ modifications in germ cells during mouse spermatogenesis. Acta Histochem Cytochem 2011;44:183-90.

45. Rousseaux S, Gaucher J, Thevenon J, Caron C, Vitte AL, Curtet $\mathrm{S}$, et al. Spermiogenesis: histone acetylation triggers male genome reprogramming. Gynecol Obstet Fertil 2009;37:519-22.

46. Meistrich ML, Mohapatra B, Shirley CR, Zhao M. Roles of transition nuclear proteins in spermiogenesis. Chromosoma 2003;111:483-8.

47. Corzett M, Mazrimas J, Balhorn R. Protamine 1: protamine 2 stoichiometry in the sperm of eutherian mammals. Mol Reprod Dev 2002;61:519-27.

48. Carrell DT, Emery BR, Hammoud S. Altered protamine expression and diminished spermatogenesis: what is the link? Hum Reprod Update 2007;13:313-27.

49. Aoki VW, Carrell DT. Human protamines and the developing spermatid: their structure, function, expression and relationship with male infertility. Asian J Androl 2003;5:315-24.

50. Shirley CR, Hayashi S, Mounsey S, Yanagimachi R, Meistrich ML. Abnormalities and reduced reproductive potential of sperm from Tnp1- and Tnp2-null double mutant mice. Biol Reprod 2004;71:1220-9.

51. Oakes CC, La Salle S, Smiraglia DJ, Robaire B, Trasler JM. A unique configuration of genome-wide DNA methylation patterns in the testis. Proc Natl Acad Sci U S A 2007;104:228-33.

52. de Mateo S, Ramos L, de Boer P, Meistrich M, Oliva R. Protamine 2 precursors and processing. Protein Pept Lett 2011;18:778-85.

53. Carrell DT, Liu L. Altered protamine 2 expression is uncommon in donors of known fertility, but common among men with poor fertilizing capacity, andmay reflect other abnormalities of spermiogenesis. J Androl 2001;22:604-10.

54. Aoki VW, Liu L, Jones KP, Hatasaka HH, Gibson M, Peterson $\mathrm{CM}$, et al. Sperm protamine 1/protamine 2 ratios are related to in vitro fertilization pregnancy rates and predictive of fertilization ability. Fertil Steril 2006;86:1408-15.

55. Torregrosa N, Dominguez-Fandos D, Camejo MI, Shirley CR, Meistrich ML, Ballesca JL, et al. Protamine 2 precursors, protamine 1/protamine 2 ratio, DNA integrity and other sperm parameters in infertile patients. Hum Reprod 2006;21:2084-9.

56. Simon L, Castillo J, Oliva R, Lewis SE. Relationships between human sperm protamines, DNA damage and assisted reproduction outcomes. Reprod Biomed Online 2011;23:724-34.

57. Huser T, Orme CA, Hollars CW, CorzettMH, Balhorn R. Raman Spectroscopy of DNA packaging in individual human sperm cells distinguishes normal from abnormal cells. J Biophotonics 2009;2:322-32.

58. Aoki VW, Emery BR, Liu L, Carrell DT. Protamine levels vary between individual sperm cells of infertile human males and correlate with viability and DNA integrity. J Androl 2006;27:890-8.

59. Nanassy L, Liu L, Griffin J, Carrell DT. The clinical utility of the protamine 1/protamine 2 ratio in sperm. Protein Pept Lett 2011;18:772-7.

60. Soteriadou KP, Remoundos MS, Katsikas MC, Tzinia AK, Tsikaris $\mathrm{V}$, Sakarellos C, et al. The Ser-Arg-Tyr-Asp region of the major surface glycoprotein of Leishmania mimics the Arg-Gly-Asp-Ser cell attachment region of fibronectin. J Biol Chem 1992;267:13980-5.

61. Haidl G, Schill WB. Assessment of sperm chromatin condensation: an important test for prediction of IVF outcome. Arch Androl 1994;32:263-6.

62. Ward WS. Function of sperm chromatin structural elements in fertilization and development. Mol Hum Reprod 2010;16:30-6.

63. Cree LH, Balhorn R, Brewer LR. Single molecule studies of DNAprotamine interactions. Protein Pept Lett 2011;18:802-10.

64. Yanagimachi R. Male gamete contributions to the embryo. Ann N Y Acad Sci 2005;1061:203-7.

65. Dominguez K, Arca CDR, Ward WS. The relationship between chromatin structure and DNA damage in mammalian spermatozoa. In: Zini A, Agarwal A, editors. Sperm chromatin: biological and clinical applications in male infertility and assisted reprod, 2011. pp.61-8.

66. Hammoud SS, Nix DA, Zhang H, Purwar J, Carrell DT, Cairns BR. Distinctive chromatin in human sperm packages genes for embryo development. Nature 2009;460:473-8.

67. Hammoud SS, Nix DA, Hammoud AO, Gibson M, Cairns BR, Carrell DT. Genome-wide analysis identifies changes in histone retention and epigenetic modifications at developmental and imprinted gene loci in the sperm of infertile men. Hum Reprod 2011;26:2558-69.

68. Arpanahi A, Brinkworth M, Iles D, Krawetz SA, Paradowska A, Platts AE, et al. Endonuclease-sensitive regions of human spermatozoal chromatin are highly enriched in promoter and CTCF binding sequences. Genome Res 2009;19:1338-49. 
69. Gan Q, Yoshida T, McDonald OG, Owens GK. Concise review: epigenetic mechanisms contribute to pluripotency and cell lineage determination of embryonic stem cells. Stem Cells 2007;25:2-9.

70. Hammoud S, Liu L, Carrell DT. Protamine ratio and the level of histone retention in sperm selected from a density gradient preparation. Andrologia 2009;41:88-94.
71. Li Y, Lalancette C, Miller D, Krawetz SA. Characterization of nucleohistone and nucleoprotamine components in the mature human sperm nucleus. Asian J Androl 2008;10:535-41.

72. Miller D, Brinkworth M, Iles D. Paternal DNA packaging in spermatozoa: more than the sum of its parts? DNA, histones, protamines and epigenetics. Reproduction 2010;139:287-301. 